\title{
Molecular Epidemiology of Early and Acute HIV Type 1 Infections in the United States Navy and Marine Corps, 2005-2010
}

\author{
Richard A. Heipertz \\ Walter Reed Army Institute of Research \\ Eric Sanders-Buell \\ Walter Reed Army Institute of Research \\ Gustavo Kijak \\ Walter Reed Army Institute of Research \\ Shana Howell \\ Walter Reed Army Institute of Research \\ Michelle Lazzaro \\ Walter Reed Army Institute of Research
}

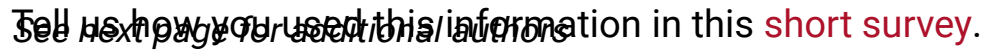

Follow this and additional works at: https://digitalcommons.unmc.edu/coph_epidem_articles

Part of the Epidemiology Commons

\section{Recommended Citation}

Heipertz, Richard A.; Sanders-Buell, Eric; Kijak, Gustavo; Howell, Shana; Lazzaro, Michelle; Jagodzinski, Linda L.; Eggleston, John; Peel, Sheila; Malia, Jennifer; Armstrong, Adam; Michael, Nelson L.; Kim, Jerome H.; O'Connell, Robert J.; Scott, Paul T.; Brett-Major, David; and Tovanabutra, Sodsai, "Molecular Epidemiology of Early and Acute HIV Type 1 Infections in the United States Navy and Marine Corps, 2005-2010" (2013). Journal Articles: Epidemiology. 99.

https://digitalcommons.unmc.edu/coph_epidem_articles/99

This Article is brought to you for free and open access by the Epidemiology at DigitalCommons@UNMC. It has been accepted for inclusion in Journal Articles: Epidemiology by an authorized administrator of DigitalCommons@UNMC.For more information, please contact digitalcommons@unmc.edu. 


\section{Authors}

Richard A. Heipertz, Eric Sanders-Buell, Gustavo Kijak, Shana Howell, Michelle Lazzaro, Linda L.

Jagodzinski, John Eggleston, Sheila Peel, Jennifer Malia, Adam Armstrong, Nelson L. Michael, Jerome H.

Kim, Robert J. O'Connell, Paul T. Scott, David Brett-Major, and Sodsai Tovanabutra 


\title{
Molecular Epidemiology of Early and Acute HIV Type 1 Infections in the United States Navy and Marine Corps, 2005-2010
}

\author{
Richard A. Heipertz Jr., Eric Sanders-Buell, ${ }^{1,2}$ Gustavo Kijak,, ${ }^{1,2}$ Shana Howell,,2 Michelle Lazzaro,,2 \\ Linda L. Jagodzinski,, John Eggleston,, ${ }^{1,2}$ Sheila Peel,, Jennifer Malia,,3 Adam Armstrong, ${ }^{4}$ \\ Nelson L. Michael, Jerome H. Kim,, Robert J. O'Connell, Paul T. Scott, \\ David M. Brett-Major, ${ }^{5,6}$ and Sodsai Tovanabutra ${ }^{1,2}$
}

\begin{abstract}
The U.S. military represents a unique population within the human immunodeficiency virus 1 (HIV-1) pandemic. The last comprehensive study of HIV-1 in members of the U.S. Navy and Marine Corps (Sea Services) was completed in 2000, before large-scale combat operations were taking place. Here, we present molecular characterization of HIV-1 from 40 Sea Services personnel who were identified during their seroconversion window and initially classified as HIV-1 negative during screening. Protease/reverse transcriptase ( $p r o / r t)$ and envelope (env) sequences were obtained from each member of the cohort. Phylogenetic analyses were carried out on these regions to determine relatedness within the cohort and calculate the most recent common ancestor for the related sequences. We identified 39 individuals infected with subtype B and one infected with CRF01_AE. Comparison of the pairwise genetic distance of Sea Service sequences and reference sequences in the env and $\mathrm{pro} / \mathrm{rt}$ regions showed that five samples were part of molecular clusters, a group of two and a group of three, confirmed by single genome amplification. Real-time molecular monitoring of new HIV-1 acquisitions in the Sea Services may have a role in facilitating public health interventions at sites where related HIV-1 infections are identified.
\end{abstract}

\section{Introduction}

$\mathbf{U}$ NAIDS RECOGNIZES MILITARY MEMBERS as a group at risk of human immunodeficiency virus 1 (HIV-1) infection $^{1}$ and the U.S. military portion of the HIV-1 pandemic is unique for several crucial reasons. Screening for anti-HIV-1 antibodies has been employed on all applicants for military service since 1985 and on active and reserve component members of the armed services since $1986 .^{2}$ In 2004, the Department of Defense instituted mandatory testing for all service members every 2 years. Testing is also conducted when clinically indicated and as related to predeployment and postdeployment processing. ${ }^{2,3}$ In addition, U.S. military personnel constitute a globally deployed risk group who are subject to unique HIV-1 risk environments and the stress of 11 years of continuous large-scale combat and support operations in support of the Global War on Terrorism and Overseas Contingency Operations, most prominently in Afghanistan and Iraq.

Several epidemiological and molecular epidemiological studies of HIV-1 have evaluated incident infections in the U.S. Army and Air Force with the most recent cohort ending in 2007. ${ }^{4-7}$ Studies have also been undertaken investigating infections in the U.S. Navy and Marine Corps, ${ }^{8-10}$ but the most recent of these considered data through May 2000, before the beginning of large-scale combat operations. Recent annual rates of infection per 1,000 HIV-1 tests range from 0.13 to 0.35 depending upon service and component (whether active or reserve force) in the Sea Services. ${ }^{2,3}$ Since the tempo of deployments and geographic breadth of missions undertaken by

${ }^{1}$ US Military HIV Research Program, Walter Reed Army Institute of Research, Silver Spring, Maryland.

${ }^{2}$ Henry M. Jackson Foundation for the Advancement of Military Medicine, Inc., Bethesda, Maryland.

${ }^{3}$ United States Public Health Service, Rockville, Maryland.

${ }^{4}$ Navy Bloodborne Infection Management Center, Navy Marine Corps Public Health Center, Bethesda, Maryland.

${ }^{5}$ Naval Medical Research Center, Silver Spring, Maryland.

${ }^{6}$ Department of Preventive Medicine and Biometrics, Uniformed Services University, Bethesda, Maryland. 
the Navy and Marine Corps in the post-9/11 era have significantly increased, important questions remain for epidemiology and prevention.

This current study aims to molecularly characterize acute and very early incident HIV-1 infections in the U.S. Navy and Marine Corps with a first known positive HIV-1 screening test between January 1, 2005 and August 3, 2010. ${ }^{3}$ The overarching public health investigation and its epidemiology have been described elsewhere. ${ }^{3}$

\section{Materials and Methods}

\section{Participants and HIV-1 testing}

The overall study was comprised of 496 participants who are enlisted U.S. Sailors and Marines with a first known positive HIV-1 screening test between January 1, 2005 and August 3, 2010. ${ }^{3}$ From January 2005 through April 2009, screening utilized the second generation enzyme-linked immunosorbent assay (ELISA) confirmed through Western blot. ${ }^{3}$ All 496 participants had their last-negative HIV-1 test rescreened using the third-generation ELISA confirmed through Western blot and nucleic acid testing (NAT) using the Abbott RealTime HIV-1 Test. The subset described in this publication is comprised of 54 participants whose last negative HIV-1 test occurred during their seroconversion window where anti-HIV-1 antibody testing was nonreactive but retrospective look back testing of repository samples detected HIV-1 nucleic acid. The seroconversion window lasts from approximately 5-19 days postinfection. ${ }^{11}$ Therefore, these 54 participants were initially misclassified as negative in their HIV-1 screening. Of the 54 samples drawn during this seroconversion window of the 496 in this cohort, 12 samples were not available because volumes were entirely consumed during HIV-1 diagnostic look back testing. Sample identification numbers given to each individual were coded to protect their personal information.

\section{Viral RNA extraction}

A volume of $0.5 \mathrm{ml}$ of plasma was extracted per sample. For samples with viral load (VL) below 10,000 copies $/ \mathrm{ml}, 1 \mathrm{ml}$ of plasma was extracted. The plasma was centrifuged for $1 \mathrm{~h}$ at $4^{\circ} \mathrm{C}$ at $20,000 \times g$. One hundred and forty microliters of plasma was left behind following the spin. The viral pellet was resuspended and extracted with the QIAamp viral RNA mini kit (Qiagen, USA) per the manufacturer's instructions.

\section{Complementary DNA (cDNA) synthesis}

Viral RNA was reverse transcribed using the SuperScript III RT kit (Invitrogen, USA) following the manufacturer's instructions. Of oligo(dT) $50 \mu \mathrm{M}$ was utilized as the primer along with kit contents diethylpyrocarbonate (DEPC) $\mathrm{H}_{2} \mathrm{O}$ and $10 \mathrm{mM}$ deoxynucleotide triphosphates (dNTP). Seven microliters of RNA was added to the mix and incubated for $5 \mathrm{~min}$ at $65^{\circ} \mathrm{C}$. A mix of $10 \times \mathrm{RT}$ buffer, DTT, $\mathrm{MgCl}_{2}$, RNase OUT, and SuperScript III RT enzyme was added to the RNA/ primer mix for a total reaction volume of $20 \mu \mathrm{l}$. The reaction proceeded for $3 \mathrm{~h}$ at $50^{\circ} \mathrm{C}$, followed by $85^{\circ} \mathrm{C}$ for $5 \mathrm{~min}$. In some cases, an internal primer (HXB2 5983-5956) was used to generate the pro/rt cDNA fragment when oligo(dT)generated cDNA was not conducive to yielding a positive pro/rt polymerase chain reaction (PCR). The cDNA obtained was used as a template for PCR at near end-point dilution. For cDNA amplification, the primers used in this study are shown in Table 1 with the PCR conditions described below.

\section{Pro/rt PCR}

The PCR for the pro/rt region (HXB2 2094-3866) utilized the AmpliTaq Gold PCR kit (Applied Biosystems, USA) per the manufacturer's instructions. Briefly, $\mathrm{MgCl}_{2}, 10 \times \mathrm{PCR}$ buffer, $\mathrm{dNTP}(1.25 \mathrm{mM})$, forward and reverse primers ( $20 \mu \mathrm{M}$ for each), and AmpliTaq Gold enzyme were made in a master mix and dilutions of cDNA were added with water to a final volume of $50 \mu \mathrm{l}$. The nested PCR strategy utilized first round PCR primers at HXB2 positions 1808-1834 and 3892-3870 and second round PCR primers at HXB2 positions 2071-2093 and 3886-3864.

\section{Env PCR}

The PCR for the env region (HXB2 5984-9063) utilized the Platinum Taq kit (Invitrogen, USA) per the manufacturer's instructions. Briefly, $10 \times$ buffer, $\mathrm{MgSO}_{4}$, dNTP $(1.25 \mathrm{mM})$, forward and reverse primers ( $20 \mu \mathrm{M}$ for each), and Platinum Taq enzyme were made in a master mix and dilutions of cDNA were added with water to a final volume of $20 \mu \mathrm{l}$. The seminested PCR strategy utilized the same forward primer in both first and second round PCR reactions and was positioned at HXB2 59585983. The first round reverse primer was positioned at HXB2 9520-9497 and the second round reverse primer at HXB2 90899064. For samples where a molecular link was observed, SGA was carried out using the same PCR protocol and the PCR products from a cut-off of $30 \%$ positive wells were used. ${ }^{12}$

Table 1. List of Primer Sequences Used for Polymerase Chain Reaction

\begin{tabular}{lll}
\hline Region (HXB2 number) & Primer/probe & Sequence $\left(5^{\prime}-3^{\prime}\right)$ \\
\hline pro/rt (2094-3866) & $\begin{array}{l}\text { Outer forward } \\
\text { Outer reverse } \\
\text { Inner forward }\end{array}$ & GGGCTTCATTAGAAGAAATGATGACAG \\
& Inner reverse & CTATTRGCTGCCCCATCTACATA \\
env (5984-9063) & Guter + inner forward & GCTGCCCCATCTACATAGAAAGT \\
& Outer reverse & TAGGCATTTCCTATGGCAGGAAGAAG \\
Full genome $(789-9,496)$ & GCAGCTGCTTATATGCAGGATCTGAGGG \\
& Outer forward & TCCAGTCCCCCCTTTCTTTTAAAAA \\
& Outer reverse & AAATCTCTAGCAGTGGCGCCCGAACAG \\
& Inner forward & GCACTCAAGGCAAGCTTTATTGAGGCTT \\
& Inner reverse & TGACTAGCGGAGGCTAGAAGGAGAGA \\
& & GCAGCTGCTTATATGCAGGATCTGAGGG
\end{tabular}




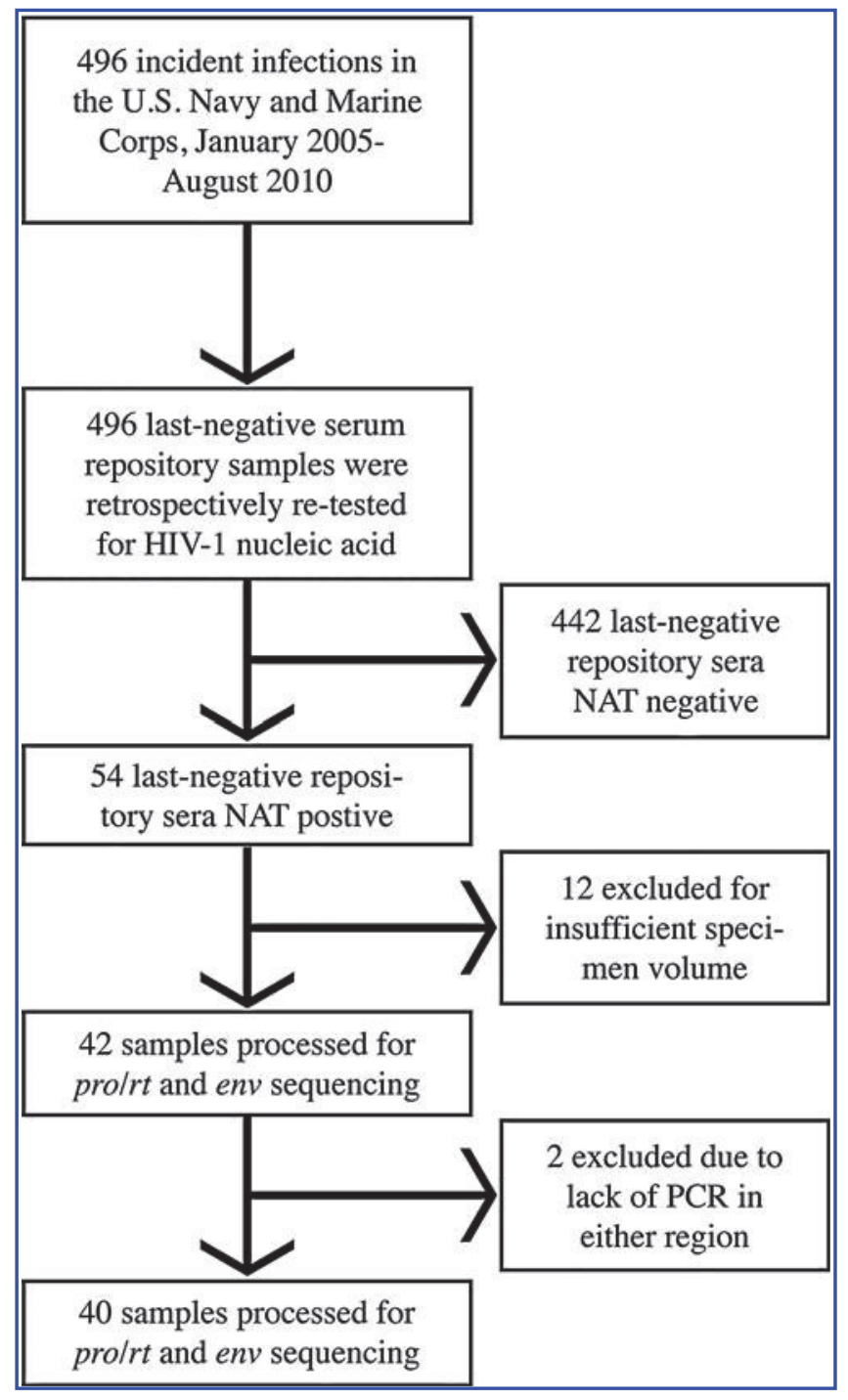

FIG. 1. Consort figure sample selection criteria. Out of the total cohort of 496 individuals with incident HIV-1 infections, 54 were misclassified as HIV-1 negative in their last negative screening. These samples were anti-HIV-1 antibody negative but nucleic acid positive leading to the misclassification of these samples.

\section{Full-genome PCR}

The PCR for full-genome sequence generation (HXB2 7899496) utilized the Advantage GC Genomic LA kit (Clontech, USA) per the manufacturer's instructions. Briefly, $2 \times$ GC-Melt LA Buffer, dNTP $(10 \mathrm{mM})$, forward and reverse primers $(20 \mu \mathrm{M}$ for each), and GC Genomic LA polymerase mix were made in a master mix and dilutions of cDNA were added with water to a final volume of $25 \mu \mathrm{l}$. The nested PCR strategy utilized first round PCR primers at HXB2 623-649 and 9632-9605 and second round PCR primers at HXB2 763-788 and 9524-9497.

\section{Sequencing}

PCR products generated by the most dilute cDNA for pro/ $r t$, env, or full-genome sequencing were purified using the PureLink Genomic DNA Purification kit (Invitrogen, USA) per the manufacturer's instructions. For PCR reactions yielding secondary or nonspecific bands, the NucleoSpin Gel and PCR Clean-up kit (Macherey-Nagel, USA) was utilized for gel extraction and purification of PCR products. Sequencing reactions of PCR products were performed using the BigDye Terminator v.3.1 Cycle Sequencing Kit (Applied Biosystems, USA) along with $5 \times$ Sequencing Buffer, and water to a final volume of $16 \mu$ l. Sequencing reactions were purified with a PCR 96 Well Kit (Princeton Separations, USA) and loaded on an Applied Biosystems 3730 DNA Analyzer.

\section{Sequence alignment}

Sequences generated from this cohort were manually edited using Sequencher 5.0 (Gene Codes Corporation, USA) and aligned using Gene Cutter (HIVDB). ${ }^{13}$ The initial HIV-1 genotype was assigned for each sequence in this cohort using the HIV-1 Genotyping Tool at the National Center for Biotechnology Information (NCBI). ${ }^{14}$ Appropriate reference sequences that covered the entire region of pro/rt, env, or full-genome were downloaded from the HIVDB and aligned. The whole alignment covering each region and consisting of sequences from this cohort plus references was manually edited using MacGDE 2.4.

\section{Phylogenetic analysis}

Reference sequences were comprised of only subtype B for the pro/rt and env regions and subtype B and CRF01_AE for the full-genome alignments. A single reference was kept per individual (earliest time point). Reference sequences with excessive " $\mathrm{N}$ " base calls and/or containing large insertions or deletions were removed from the analysis. Alignments for $\mathrm{pro} / \mathrm{rt}$, env, and full-genome regions were phylogenetically analyzed using Molecular Evolutionary Genetics Analysis (MEGA) 5.0. ${ }^{15}$ In all regions, subtype B references belonging to Thai B, termed B', were excluded from analysis since sequences from this cohort did not belong to $\mathrm{B}^{\prime}$. In the pro/rt region, 144 nucleotide positions were removed from analysis due to their association with drug resistance that may confound the analysis. This comprehensive list of drug resistance sites was taken from the Stanford University HIV Drug Resistance Database. Maximum likelihood (ML) analysis was utilized to phylogenetically categorize the pro/rt and env regions. The ML method used the General Time Reversible (GTR) substitution model and gaps were treated as partial deletion with a cutoff of $50 \%$ of site coverage. Neighborjoining analysis with the Kimura two-parameter model of substitution ${ }^{16}$ was utilized to determine the subtype of the fullgenome sequences. Gaps were also treated as partial deletion with a cutoff of $50 \%$ of site coverage. Phylogenetic trees generated from the analyses were visualized using FigTree 1.3.1.

\section{Pairwise genetic distance analysis}

Calculations for pairwise genetic distance of env and $\mathrm{pro} / \mathrm{rt}$ regions were performed in MEGA 5.0. ${ }^{15}$ The Kimura twoparameter substitution model ${ }^{16}$ was utilized with partial deletion $(50 \%)$ of gaps. The distance matrix data were analyzed using JMP Genomics Software 4.0 (SAS Institute Inc., USA).

\section{Inferring most recent common ancestor (MRCA) using Bayesian Markov chain Monte Carlo (MCMC) relaxed molecular clock}

To determine the time to the MRCA, we used a Bayesian MCMC-based approach. BEAST version 1.6.2 was used for 
FIG. 2. Maximum likelihood (ML) phylogenetic trees generated from pro/rt and env sequencing. ML phylogenetic tree of the pro/rt region (HXB2 2094-3866) from 1,296 subtype B reference sequences and 39 sequences from this cohort are shown (A). Five hundred and three subtype B references and 38 sequences from this cohort were analyzed by ML and covered the env region (HXB2 5984-9063) (B). The five samples shown to be part of a cluster were further sequenced to generate 10 env SGA sequences per sample. Red *, cohort sequences; green *, cohort sequences part of the dual cluster; blue *, cohort sequences part of the tri cluster; black box, indicates dual quasispecies.

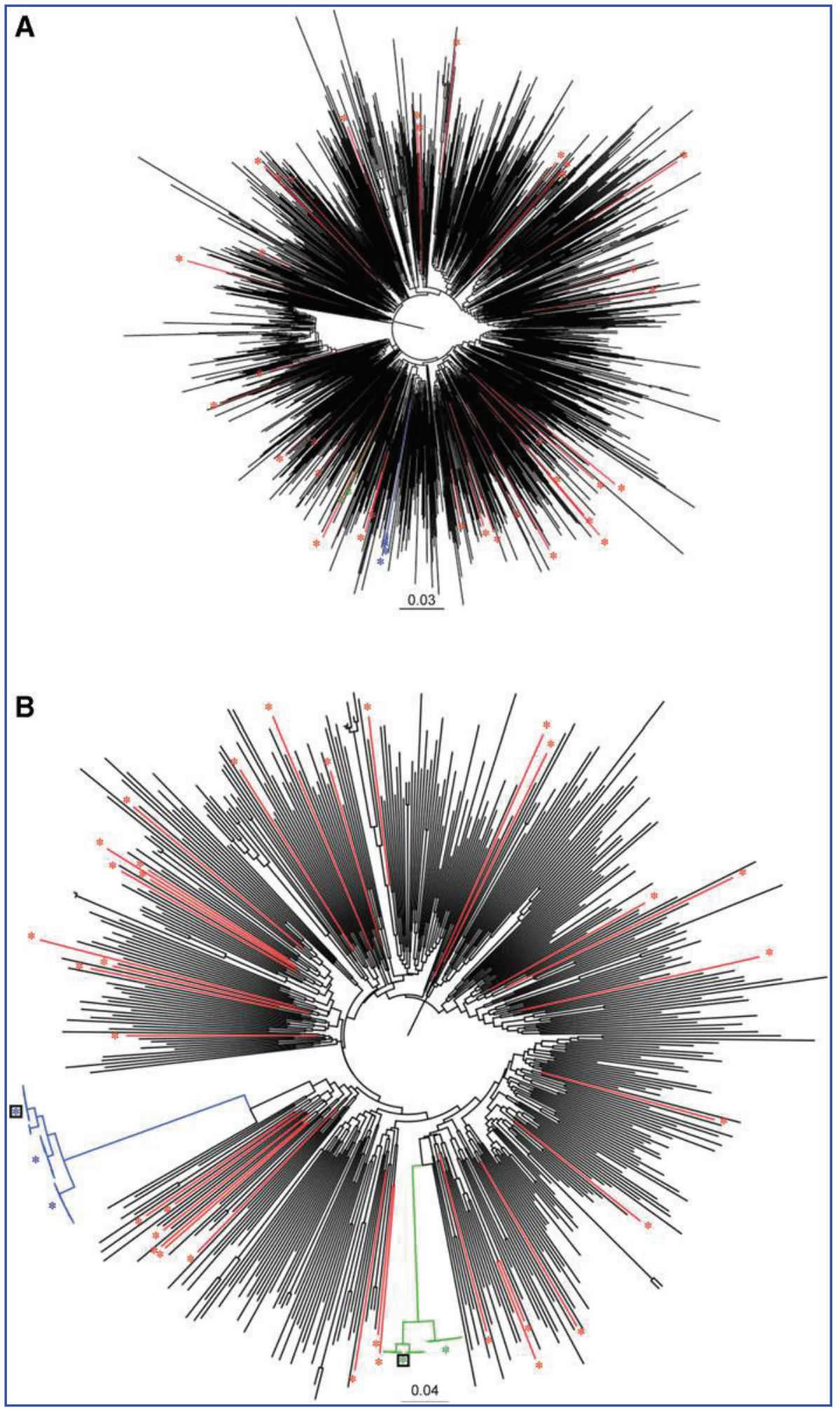

all calculations. ${ }^{17}$ SGA sequences generated from the same individual were grouped into a single taxon to allow for calculations. Analyses were carried out using the GTR substitution model and a relaxed uncorrelated exponential clock $^{17,18}$ with exponential growth prior. The MRCA calculations reported were based on an ESS of greater than 300 via Tracer version 1.5. Trees generated from the BEAST runs were visualized after $10 \%$ burn-in with TreeAnnotator version 1.6.2. A graph of the MRCA with 95\% confidence intervals was generated using Prism 5.0 (GraphPad Software, USA).

\section{GenBank submission}

The sequences from this study have been submitted to GenBank under the numbers JX863919-JX864047. 


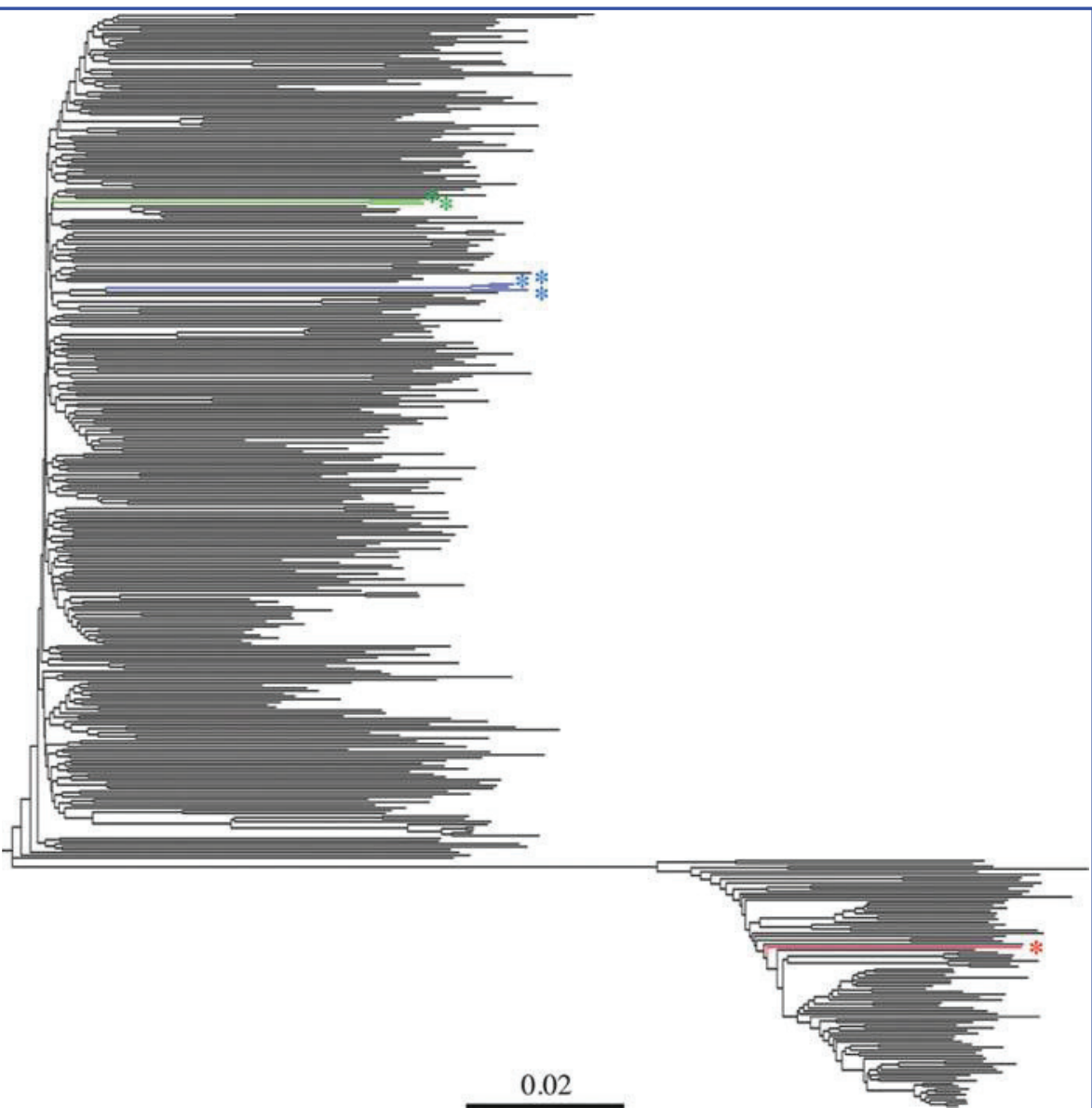

FIG. 3. Neighbor-joining tree generated from full-genome sequencing. An alignment was generated covering all gene-coding regions of the HIV-1 genome (HXB2 789-9496). A total of 299 subtype B reference sequences was compared alongside the five linked samples from this cohort. Additionally, 88 CRF01_AE references and the single CRF01_AE sequences from this cohort are represented. Red *, CRF01_AE cohort sequence; green *, cohort sequences part of the dual cluster; blue *, cohort sequences part of the tri cluster.

\section{Results}

\section{Participants}

The Sea Services study identified 496 HIV seroconversions ${ }^{3}$ and look back testing of the last seronegative sample in each of these cases showed that 54 were positive by NAT. Twelve of 54 samples were not available because volumes were entirely consumed during HIV-1 diagnostic look back testing. Additionally, two samples were not amplifiable either in protease/reverse transcriptase ( pro/rt) or envelope (env) and one sample was amplifiable only in pro/rt (Fig. 1). The collection dates ranged from April 5, 2002 to December 21, 2009. The mean VL for these samples was 6.8 with a range of 3.7 to $7.8 \log$ copies per ml.

\section{HIV-1 subtype designation and cluster identification}

Based on sequenced regions of pro/rt and env, 39 of the 40 samples were subtype B while one was CRF01_AE. None of the subtype B sequences clustered with Thai B, so-called B'. We further examined the genetic diversity of the subtype $B$ sequences with other subtype B reference sequences obtained from the LANL HIVDB. ${ }^{13}$ Given that sequences in our cohort were not classified as B', B' sequences were excluded from our analysis.

Thirty-nine sequences from our cohort (red *) and 2,296 pro/rt subtype B references were analyzed by ML (Fig. 2A). Prior to tree construction, 144 nucleotide positions (48 amino acids) corresponding to potential drug resistance were considered potentially confounding and were removed from analysis. Most of the sequences of interest were dispersed throughout the subtype B reference sequences. However, there was a group of two samples (green *) [306159, 306512] and a group of three samples (blue *) [306344, 306376, 306517] that formed tight monophyletic clusters.

A similar analysis approach was performed on the env sequences. Thirty-eight sequences from our cohort and 538 reference sequences were analyzed in env by the ML method, confirming the subtypes identified in pro/rt (Fig. 2B). Figure $2 \mathrm{~B}$ also shows an even distribution of our cohort samples $\left(\mathrm{red}^{*}\right)$ throughout the reference sequences. We have employed SGA, a powerful technique that allows the fine analysis of sequence differences. It has been particularly useful in the analysis of transmitted founder viruses and in the evaluation of breakthrough infections in vaccine studies. ${ }^{19,20}$ SGA of $e n v$ was performed on the five molecularly linked samples. The generation of multiple sequences from the same patient for env SGA further strengthens the link within each cluster and also reveals that 306159 and 306344 have two predominant quasispecies (green or blue * surrounded by a black box) that were also confirmed by visual inspection and HIVDB Highlighter Plot (data not shown). ${ }^{13}$

Furthermore, we obtained full-genome sequences (HXB2 789-9496) of the five samples from these two linked clusters as well as the single CRF01_AE sequence. Two hundred and ninety-nine subtype B and 88 CRF01_AE reference sequences 
FIG. 4. Pairwise genetic distance calculations for the env region. The pairwise genetic distance for the env region was calculated for reference and cohort sequences using the Kimura two-parameter model. For each figure section, the mean pairwise genetic distance is annotated in the center of each graph. (A) A comparison of references and sequences from this cohort; (B) cohort sequences compared to references identified prior to the year 2000; (C) cohort sequences compared to references identified from 2000 to 2004; (D) cohort sequences compared to references identified from 2005 to the present; (E) cohort sequence comparison without any references.

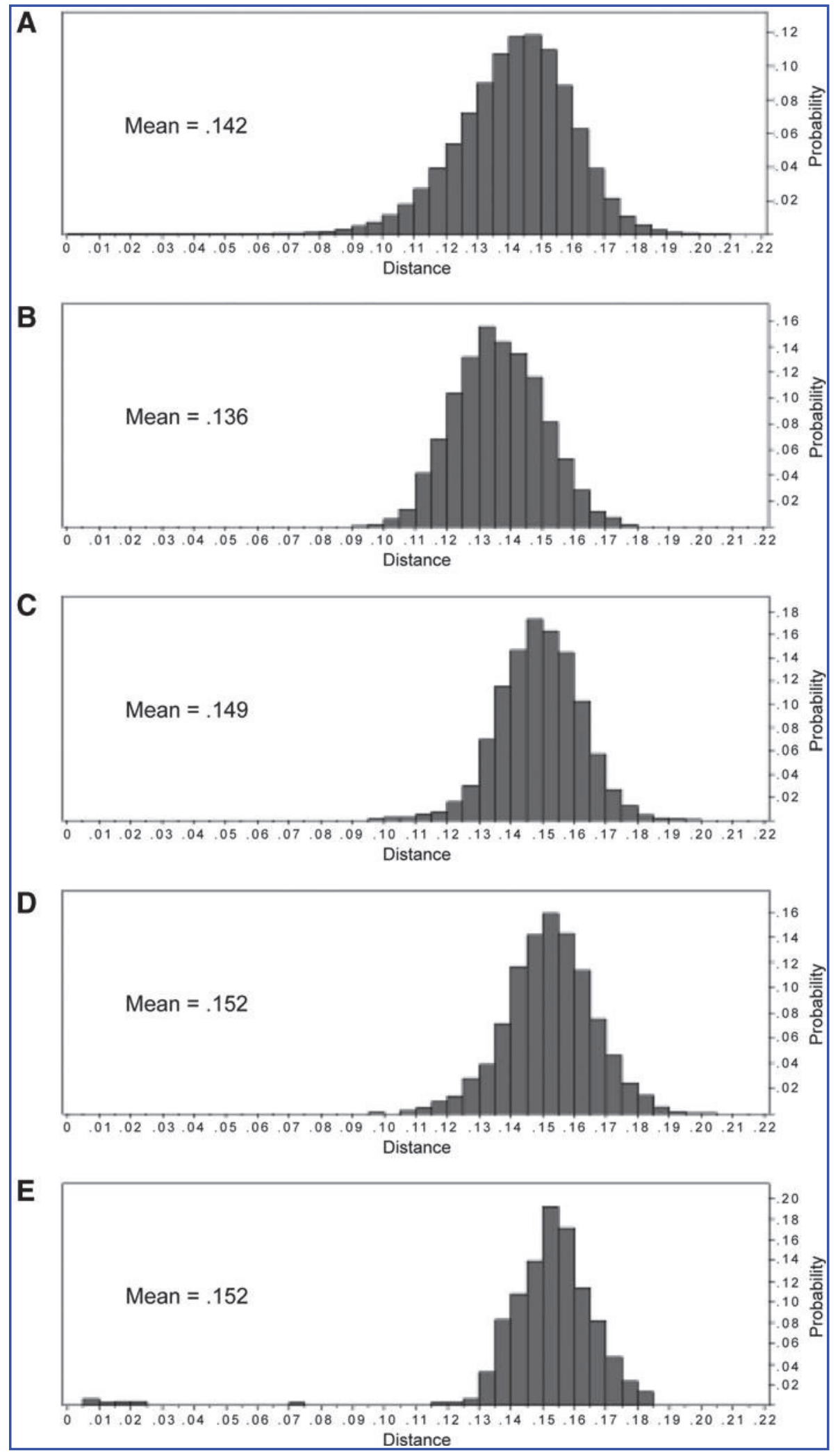

from the HIVDB were included in the neighbor-joining phylogenetic analysis. In concordance with our previous data, the clusters were readily apparent over the full genome, and the CRF01_AE sequence clustered with other CRF01_AE reference sequences identified from Southeast Asia (Fig. 3).

\section{Genetic distance calculations of molecularly linked samples}

We further characterized the relationship of the two molecularly linked groups through comparison of sequence 
diversity of the two groups to the reference sequences, stratified by the following years: pre-2000, 2000-2004, and 2005present. Reference sequences that did not have a date were removed from this analysis.

Initially, we considered the env region since it is more diverse than pro/ $r$, thereby providing a greater likelihood to observe differences between our linked samples and others from the cohort. For the three sequences suspected to be molecularly linked and having a single quasispecie, we generated a consensus sequence of the 10 env SGA obtained per individual and used each consensus for analysis of the genetic distance. For the two sequences with dual quasispecies, a separate consensus was generated for each quasispecie such that each of these dually infected individuals would be represented by two consensus sequences in the analysis. We chose to use consensus env sequences in the analysis because we did not want to overemphasize the signal observed if each of the SGA sequences was used separately. The overall comparison of Sea Service samples plus reference sequences showed a mean genetic distance of 0.142 (Fig. 4A). Interestingly, when sequences from this cohort were compared to the references characterized as pre2000, 2000-2004, and 2005-present, a significant increase in genetic distance was observed from 0.136 to 0.149 to 0.152 ( $p$-values $<0.0001$ ), respectively (Fig. 4B-D). Additionally, the comparison of cohort sequences to each other, without references, yielded a mean genetic distance of 0.152 (Fig. 4E). A comparison of the genetic distances of the consensus sequences from each member of the cluster is shown in Table 2 (top portion). The genetic distance of sequences in the two molecular clusters was less than 0.025 and well outside of the bell curve distribution observed in all comparisons, providing further evidence of the molecular link between these groups of sequences.

After removal of nucleotide positions associated with drug resistance, comparison of cohort and reference sequences in the $\mathrm{pro} / \mathrm{rt}$ region showed a mean genetic distance of 0.066 (Fig. 5A). In parallel with env data, cohort samples from pre-2000, 2000-2004, and 2005-present showed a significant increase in genetic distance from 0.059 to 0.066 to 0.069 ( $p$-values $<0.0001)$, respectively (Fig. 5B-D). Through comparison between these cohort sequences, the same genetic distance of 0.069 was observed for reference sequences over the same time period (Fig. 5E). A comparison of the genetic distances of the consensus sequences from each member of the cluster is shown in Table 2 (bottom portion). The genetic distances of sequences in the two molecular clusters were less than 0.01 and fall outside the bell curve distribution.

\section{Dating of linked molecular clusters}

We used the Bayesian MCMC approach to calculate time to the MRCA of each linked sample and the overall time to MRCA for each cluster. All 10 env SGA sequences generated per individual were used in calculations and grouped as a single entity. The draw dates for each sample were used in the MRCA calculation. Additionally, the last negative test dates (4-18 months, see Table 3 ) were used as a guide to verify the MRCA calculations produced in the analysis.

With the dual cluster, 306159 and 306512, there was a large period of time between the two samples (Fig. 6A and Table 3). Sample 306512 had a mean MRCA calculation of 0.58 months
Table 2. Pairwise Genetic Distance OF THE LiNKED GROUPS

$306159 \_b \quad 306512 \quad 306344 \_b \quad 306376 \quad 306517$

\begin{tabular}{|c|c|c|c|c|c|}
\hline \multicolumn{6}{|l|}{ Env } \\
\hline 306159_a & 0.008 & 0.024 & \multirow{5}{*}{0.008} & \multirow{5}{*}{$\begin{array}{l}0.009 \\
0.010\end{array}$} & \\
\hline 306159_b & & 0.024 & & & \\
\hline 306344_a & & & & & 0.018 \\
\hline 306344_b & & & & & 0.017 \\
\hline 306376 & & & & & 0.014 \\
\hline \multicolumn{6}{|l|}{ Pro/rt } \\
\hline 306159 & & 0.006 & & & \\
\hline 306344 & & & & 0.003 & 0.009 \\
\hline 306376 & & & & & 0.008 \\
\hline
\end{tabular}

The two linked groups and the pairwise genetic distance of the consensus sequence of each sample within the group are shown. The top portion of the table shows env distances and the bottom portion shows $\mathrm{pro} / \mathrm{rt}$ distances.

(95\% upper-lower of 2.0-0.02) while that for 306159 was 53.5 months (95\% upper-lower of 56.83-52.05) prior to the 306512 collection date (Fig. 6B). The MRCA of this dual cluster was calculated to be 56.1 months (95\% upper-lower of 65.4152.06), in agreement with the last known negative HIV-1 test for each individual (Table 3).

The cluster of three sequences, 306344, 306376, and 306517, had a shorter time period between samples, including two test dates within 3 months (Fig. 6C and Table 3). Sequence 306517 had a mean MRCA of 0.47 (95\% upper-lower of 1.26-0.04), 306376 of 30.38 (95\% upper-lower of 30.94-30.03), and 306344 of 34.32 months (95\% upper-lower of 36.34-33.16) prior to the 306517 collection date (Fig. 6D). Overall, this cluster had an MRCA date of 34.41 months (95\% upper-lower of 36.4933.18), consistent with the last known negative HIV-1 test (Table 3).

\section{Discussion}

Using samples from enlisted U.S. Navy and Marine Corps personnel whose first known positive HIV-1 screening test occurred between January 1, 2005 and August 3, 2010, we characterized sequences from 40 of 54 cohort members with samples drawn in the seroconversion window. Non-B subtypes, including recombinant viruses, have been identified previously in U.S. military cohorts. ${ }^{7-10}$ In this study, all but one sequence in this cohort was subtype B, suggesting that 11 years of continuous large-scale combat and support operations has not significantly altered the HIV-1 subtypes previously reported to be circulating in Sea Services personnel. ${ }^{8-10}$

The sequencing of two regions of the HIV-1 genome increased the likelihood of correct subtype designation. While linkage analysis did not change in this study from the use of two regions, others have used a similar strategy of sequencing multiple HIV-1 genes and have found this to be an effective tool to characterize transmission groups. ${ }^{21-25}$ The use of ML phylogenetic analysis was one method used to identify transmission clusters, but we also employed the calculation of the pairwise genetic distance over the pro/rt and env regions. ${ }^{22}$ Comparing the genetic distance of our linked samples to all subtype B reference sequences spanning the same regions, we observed that the genetic distance of our linked samples was below the mean distance of all reference 
FIG. 5. Pairwise genetic distance calculations for the pro/rt region. The pairwise genetic distance for the pro/rt region was calculated for reference sequences and cohort sequences using the Kimura two-parameter model. For each figure section, the mean pairwise genetic distance is annotated in the center of each graph. (A) A comparison of references and cohort sequences; (B) cohort compared to references identified prior to the year 2000; (C) cohort sequences compared to references identified from 2000 to 2004; (D) cohort sequences compared to references identified from 2005 to the present; (E) cohort sequence comparison without any references.
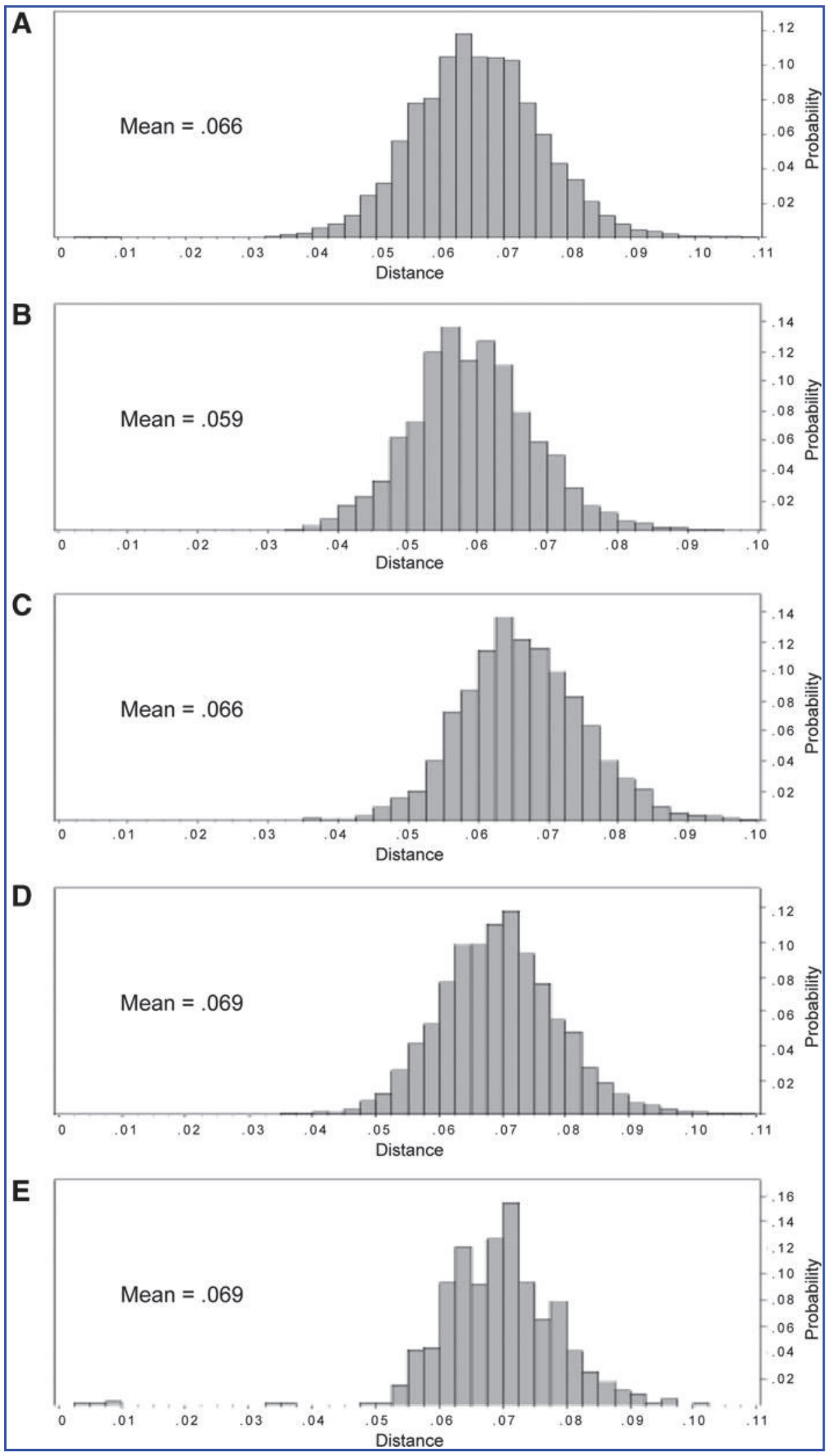

sequences compared. Our group of two (306159 and 306512) and three (306344, 306376, and 306517) linked samples was separated by years between diagnoses. Samples 306159 and 306512 are separated by more than 4 years while 306344 and 306376 are separated by 3 months and 306376 and 306517 by more than 2 years. These data are consistent with the findings of Redd et al., ${ }^{26}$ who reported that transmitted viruses more closely resemble donor archival sequences than the diverse quasispecies observed in the donor prior to transmission. This further supports the idea that unique features of transmitted 
TABle 3. Dates of Importance for the LinKed SAmples

\begin{tabular}{lccc}
\hline $\begin{array}{l}\text { Spin } \\
\text { number }\end{array}$ & $\begin{array}{c}\text { Last } \\
\text { negative } \\
\text { test date }\end{array}$ & $\begin{array}{c}\text { First } \\
\text { positive } \\
\text { test date }\end{array}$ & $\begin{array}{c}\text { Calculated } \\
\text { HIV-1 } \\
\text { acquisition date }\end{array}$ \\
\hline 306159 & 24-Feb-2004 & 14-Apr-2005 & 08-Mar-2005 \\
306512 & 15-Apr-2009 & 20-Aug-2009 & 03-Aug-2009 \\
306344 & 18-May-2006 & 09-Mar-2007 & 12-Feb-2007 \\
306376 & 14-Dec-2005 & 04-Jun-2007 & 30-May-2007 \\
306517 & 22-Aug-2009 & 21-Dec-2009 & 07-Dec-2009 \\
\hline
\end{tabular}

Important dates associated with the five samples demonstrated to be molecularly linked. The last negative HIV-1 test date for each individual is given along with the first positive test date, which is the collection date of the sequences described here. The approximate acquisition date based on Bayesian MCMC analysis is also shown.

founder viruses are required to overcome the transmission bottleneck. ${ }^{27,28}$

Even in the setting of short transmission periods, distinguishing between common or linked sources is problematic. ${ }^{29,30}$ To determine direction of transmission through these methods would require multiple samples taken before and after infection for each individual in the transmission cluster. ${ }^{29}$ This is not feasible in a force-wide screening program. A convenience survey of a subgroup of this cohort revealed several self-identified risk factors including multiple and varied sexual exposures during the risk window, ${ }^{31}$ further complicating any such determination. However, these methods combined with conventional epidemiologic approaches could provide strong transmission information relevant to public health.

Bayesian MCMC calculation for the time to the MRCA of each sample agrees with the initial date of diagnosis regarding which individual was diagnosed before another in each cluster. It is not known if others are involved in each network/ cluster, either from the larger group of samples from this cohort that were not analyzed ${ }^{3}$ or part of the civilian population that interacts with this cohort. In addition, two samples, 306196 and 306501, whose genetic distance calculations to each other were relatively low, 0.074 for $e n v$ and 0.035 for pro / $r t$, were characterized as unlinked because the genetic distance between the two sequences in env and pro/ $r$ fall within the bell curve distribution of all reference sequences for each

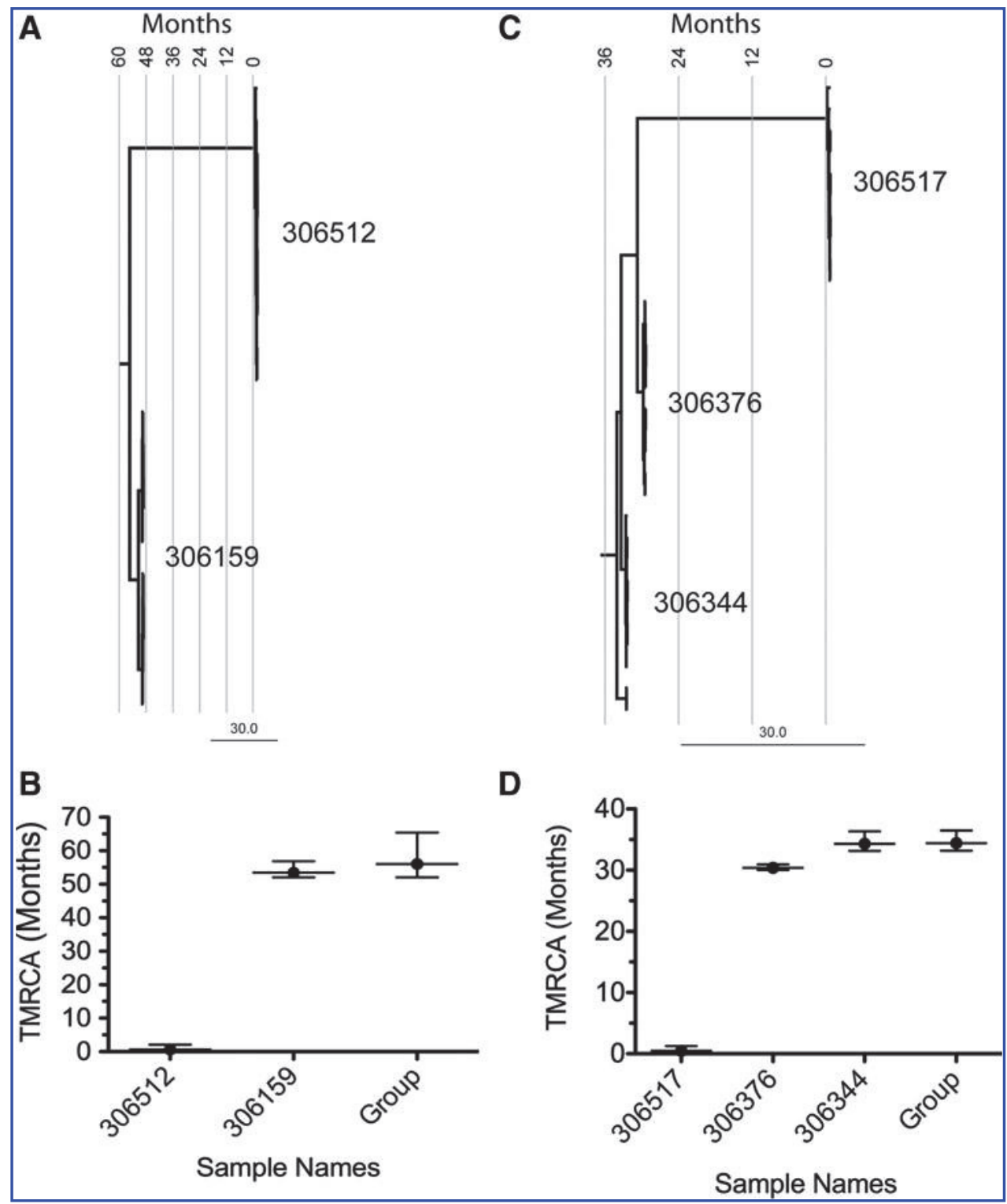

FIG. 6. Relaxed-clock use for cluster analysis. The most recent common ancestor (MRCA) was calculated for each of the linked clusters using a Bayesian Markov chain Monte Carlo (MCMC)-based approach on the env region. The date of sample collection was used in the calculation of the MRCA. The dual cluster (306159, 306512) MCMC-based consensus tree is calibrated to the time prior to the 306512 collection date (A). A graphic representation, including the upper and lower $95 \%$ confidence interval for MRCA of each sample and the cluster as a whole, is shown (B). The tri cluster (306344, 306376, 306512) MCMC-based consensus tree is calibrated to the time prior to the 306517 collection date (C). A graphic representation, including the upper and lower 95\% confidence interval for MRCA of each sample and the cluster as a whole, is shown (D). 
region, as shown in Figs. $4 \mathrm{~A}$ and $5 \mathrm{~A}$. A bridging sample was not identified in this subgroup of seroconversion, earlyincident infections within this cohort.

A second observation made during this ancestor analysis was that the mean genetic distance of sequences from this cohort compared to reference sequences increased over the time period evaluated: pre-2000, 2000-2004, and 2005-present (Figs. 4B-D and 5B-D, respectively). Previous studies reported that HIV-1 genetic diversity increases by approximately $1 \%$ per year ${ }^{32,33}$ due to its high mutation rate. ${ }^{34}$ Therefore, it is not unexpected that HIV-1 genetic diversity within subtype B increases over time. In fact, others have made similar observations with HIV-1 subtype B and C through comparison of the gag and env genes. ${ }^{35}$ Taken together with previous observations regarding HIV-1 diversity increase over time, acquired subtype B HIV-1 in this cohort continues to be part of the larger HIV-1 epidemic rather than comprising an isolated network of transmitted viruses (compare Figs. 4D and $\mathrm{E}$ and $5 \mathrm{D}$ and $\mathrm{E})$.

Among these 40 analyzed sequences, five (12.5\%) were linked. While patients identified in the seroconversion window may differ from the overall cohort for a number of reasons including risk behavior prompted self-referral for testing, extrapolating to the entire cohort of Sailors and Marines in the parent public health investigation, as many as 62 individuals may have acquired linked HIV-1 viruses. While this would represent a minority of new HIV-1 infections in this cohort, minimizing the number of individuals who are part of transmission clusters is essential. More analysis is needed to incorporate chronic infections in such analyses, particularly ones identified in similar time frames and at the same locations as seroconverting infections. This would allow more robust mapping and characterization of transmission networks to include identification of persistent, common transmission nodes.

Previous studies have shown that HIV-1 transmission outbreaks often occur over a limited time frame $e^{21,30,36}$ and early identification of transmission groups would aid in the design of specific public health interventions to prevent future spread. A previous publication highlighted the use and potential positive impact of molecular surveillance on control of HIV-1 spread within targeted populations. ${ }^{37}$ While care must be taken to protect personal confidentiality, real-time identification of transmission networks within the U.S. military may enhance public health interventions against HIV-1 transmission in the force and is an area of need for further study. Realtime molecular surveillance of HIV-1 acquisition in the U.S. military should be evaluated for its potential public health impact.

\section{Acknowledgments}

The authors would like to thank Dr. Angie Eick and the Department of Defense Serum Repository for reconciling and pulling the archived serum samples. We would also like to thank retired Navy Captain Neal Naito when at the Bureau of Medicine and Surgery who assisted in obtaining financial sourcing for this effort and Dr. Shilpa Hakre and Mr. Mark Milazzo of the U.S. Military HIV Research Program, Department of Epidemiology and Threat Assessment who assisted with force testing data management and interpretation. Finally, we would like to thank Taylor Cervenka, Holly Hack,
Janelle Kuroiwa, Annemarie O'Sullivan, and Laurin Stevenson for their technical support.

This project was funded in part by the U.S. Navy Bureau of Medicine and Surgery and the Military Infectious Diseases Research Program, project MIDRP-H014010OTPPOC. These studies were supported by a cooperative agreement (W81XWH-07-2-0067) between the Henry M. Jackson Foundation for the Advancement of Military Medicine and the Department of Defense.

The purpose of this research is to inform public health decisions regarding force health protection of U.S military service members. The results obtained from this study will in no way be used in a criminal setting for any of the individuals described. This public health activity was reviewed by the Walter Reed Army Institute of Research (WRAIR) Institutional Review Board and termed RV314. The views expressed in this article are those of the authors and do not reflect the official policy or position of the Departments of the Army, Navy, or Defense, the Uniformed Services University, or any agency of the U.S. Government. Mention of trade names, commercial products, or organizations does not imply endorsement by the U.S. government.

\section{Author Disclosure Statement}

No competing financial interests exist.

\section{References}

1. Feldbaum H, Lee K, and Patel P: The national security implications of HIV/AIDS. PLoS Med 2006;3(6):e171.

2. Updates: Routine screening for antibodies to HIV-1, civilian applicants for U.S. military service and U.S. Armed Forces, active and reserve components. MSMR 2011;18(8):15-22.

3. Brett-Major DM, Hakre S, Naito NA, et al.: Epidemiology of contemporary seroincident HIV infection in the Navy and Marine corps. Military Med 2012;177(11):1328-1334.

4. Scott PT, Hakre S, Myles O, et al.: Investigation of incident HIV infections among U.S. Army soldiers deployed to Afghanistan and Iraq, 2001-2007. AIDS Res Hum Retroviruses 2012;28(10):1308-1312.

5. Hakre S, Brett-Major DM, Singer DE, et al.: Medical encounter characteristics of HIV seroconverters in the US Army and Air Force, 2000-2004. J Acquir Immune Defic Syndr 2011;56(4):372-380.

6. Bautista CT, Sateren WB, Sanchez JL, et al.: HIV incidence trends among white and African-American active duty United States Army personnel (1986-2003). J Acquir Immune Defic Syndr 2006;43(3):351-355.

7. Singer DE, Bautista CT, O'Connell RJ, et al.: HIV infection among U.S. Army and Air Force military personnel: Sociodemographic and genotyping analysis. AIDS Res Hum Retroviruses 2010;26(8):889-894.

8. Brodine SK, Shaffer RA, Starkey MJ, et al.: Drug resistance patterns, genetic subtypes, clinical features, and risk factors in military personnel with HIV-1 seroconversion. Ann Intern Med 1999;131(7):502-506.

9. Brodine SK, Starkey MJ, Shaffer RA, et al.: Diverse HIV-1 subtypes and clinical, laboratory and behavioral factors in a recently infected US military cohort. AIDS 2003;17(17):2521-2527.

10. Tovanabutra S, Brodine SK, Mascola JR, et al.: Characterization of complete HIV type 1 genomes from non-B subtype infections in U.S. military personnel. AIDS Res Hum Retroviruses 2005;21(5):424-429. 
11. Fiebig EW, Wright DJ, Rawal BD, et al.: Dynamics of HIV viremia and antibody seroconversion in plasma donors: Implications for diagnosis and staging of primary HIV infection. AIDS 2003;17(13):1871-1879.

12. Salazar-Gonzalez JF, Bailes E, Pham KT, et al.: Deciphering human immunodeficiency virus type 1 transmission and early envelope diversification by single-genome amplification and sequencing. J Virol 2008;82(8):3952-3970.

13. LANL: HIV Sequence Database. HIVDB. Available at www.hiv.lanl.gov/. Accessed June 8, 2013.

14. Rozanov M, Plikat U, Chappey C, Kochergin A, and Tatusova T: A web-based genotyping resource for viral sequences. Nucleic Acids Res 2004;32(Web Server issue):W654-659.

15. Tamura K, Peterson D, Peterson N, Stecher G, Nei M, and Kumar S: MEGA5: Molecular evolutionary genetics analysis using maximum likelihood, evolutionary distance, and maximum parsimony methods. Mol Biol Evol 2011;28(10):2731-2739.

16. Kimura M: A simple method for estimating evolutionary rates of base substitutions through comparative studies of nucleotide sequences. J Mol Evol 1980;16(2):111-120.

17. Drummond AJ and Rambaut A: BEAST: Bayesian evolutionary analysis by sampling trees. BMC Evol Biol 2007;7:214.

18. Drummond AJ, Ho SY, Phillips MJ, and Rambaut A: Relaxed phylogenetics and dating with confidence. PLoS Biol 2006;4(5):e88.

19. Rolland M, Edlefsen PT, Larsen BB, et al.: Increased HIV-1 vaccine efficacy against viruses with genetic signatures in Env V2. Nature 2012;490(7420):417-420.

20. Rolland M, Tovanabutra S, deCamp AC, et al.: Genetic impact of vaccination on breakthrough HIV-1 sequences from the STEP trial. Nat Med 2011;17(3):366-371.

21. Lewis F, Hughes GJ, Rambaut A, Pozniak A, and Leigh Brown AJ: Episodic sexual transmission of HIV revealed by molecular phylodynamics. PLoS Med 2008;5(3):e50.

22. Campbell MS, Mullins JI, Hughes JP, et al.: Viral linkage in HIV-1 seroconverters and their partners in an HIV-1 prevention clinical trial. PLoS One 2011;6(3):e16986.

23. Han Z, Leung TW, Zhao J, et al.: A HIV-1 heterosexual transmission chain in Guangzhou, China: A molecular epidemiological study. Virol J 2009;6:148.

24. Ragonnet-Cronin M, Ofner-Agostini M, Merks $\mathrm{H}$, et al.: Longitudinal phylogenetic surveillance identifies distinct patterns of cluster dynamics. J Acquir Immune Defic Syndr 2010;55(1):102-108.

25. English S, Katzourakis A, Bonsall D, et al.: Phylogenetic analysis consistent with a clinical history of sexual transmission of HIV-1 from a single donor reveals transmission of highly distinct variants. Retrovirology 2011;8:54.

26. Redd AD, Collinson-Streng $\mathrm{AN}$, Chatziandreou $\mathrm{N}$, et al:: Previously transmitted HIV-1 strains are preferentially selected during subsequent sexual transmissions. J Infect Dis 2012;206(9):1433-1442.
27. Whitney JB, Hraber PT, Luedemann C, et al.: Genital tract sequestration of SIV following acute infection. PLoS Pathog 2011;7(2):e1001293.

28. Salazar-Gonzalez JF, Salazar MG, Keele BF, et al.: Genetic identity, biological phenotype, and evolutionary pathways of transmitted/founder viruses in acute and early HIV-1 infection. J Exp Med 2009;206(6):1273-1289.

29. Pillay D, Rambaut A, Geretti AM, and Brown AJ: HIV phylogenetics. BMJ 2007;335(7618):460-461.

30. Pilcher CD, Wong JK, and Pillai SK: Inferring HIV transmission dynamics from phylogenetic sequence relationships. PLoS Med 2008;5(3):e69.

31. Hakre S, Armstrong AW, O'Connell R J, Michael NL, Scott PT, and Brett-Major DM: A pilot online survey assessing risk factors for HIV acquisition in the Navy and Marine Corps, 2005-2010. J Acquir Immune Defic Syndr 2012;61(2):125130.

32. Herbeck JT, Nickle DC, Learn GH, et al.: Human immunodeficiency virus type 1 env evolves toward ancestral states upon transmission to a new host. J Virol 2006;80(4):1637-1644.

33. Shankarappa R, Margolick JB, Gange SJ, et al.: Consistent viral evolutionary changes associated with the progression of human immunodeficiency virus type 1 infection. J Virol 1999;73(12):10489-10502.

34. Gao F, Chen Y, Levy DN, Conway JA, Kepler TB, and Hui $\mathrm{H}$ : Unselected mutations in the human immunodeficiency virus type 1 genome are mostly nonsynonymous and often deleterious. J Virol 2004;78(5):2426-2433.

35. Woo J, Robertson DL, and Lovell SC: Constraints on HIV-1 diversity from protein structure. J Virol 2010;84(24):1299513003.

36. Hughes GJ, Fearnhill E, Dunn D, Lycett SJ, Rambaut A, and Leigh Brown AJ: Molecular phylodynamics of the heterosexual HIV epidemic in the United Kingdom. PLoS Pathog 2009;5(9):e1000590.

37. Smith DM, May SJ, Tweeten S, et al.: A public health model for the molecular surveillance of HIV transmission in San Diego, California. AIDS 2009;23(2):225-232.

Address correspondence to: Sodsai Tovanabutra Viral Genetics Section Laboratory of Molecular Virology and Pathogenesis U.S. Military HIV Research Program and the Henry M. Jackson Foundation for the Advancement of Military Medicine Walter Reed Army Institute of Research 503 Robert Grant Avenue, Room 2N25 Silver Spring, Maryland 20910

E-mail: stovanabutra@hivresearch.org 


\section{This article has been cited by:}

1. Gerald K. Chege, Craig H. Adams, Alana T. Keyser, Valerie Bekker, Lynn Morris, Francois J. Villinger, Anna-Lise Williamson, Rosamund E. Chapman. 2021. Infection of Chinese Rhesus Monkeys with a Subtype C SHIV Resulted in Attenuated In Vivo Viral Replication Despite Successful Animal-to-Animal Serial Passages. Viruses 13:3, 397. [Crossref]

2. Yuruo Li, Hongjie Liu, Habib O. Ramadhani, Nicaise Ndembi, Trevor A. Crowell, Gustavo Kijak, Merlin L. Robb, Julie A. Ake, Afoke Kokogho, Rebecca G. Nowak, Charlotte Gaydos, Stefan D. Baral, Erik Volz, Sodsai Tovanabutra, Man Charurat. 2020. Genetic clustering analysis for HIV infection among MSM in Nigeria: implications for intervention. AIDS 34:2, 227-236. [Crossref]

3. Erik Billings, Gustavo H. Kijak, Eric Sanders-Buell, Nicaise Ndembi, Anne Marie O'Sullivan, Sylvia Adebajo, Afoke Kokogho, Mark Milazzo, Kara Lombardi, Stefan Baral, Rebecca Nowak, Habib Ramadhani, Robert Gramzinski, Merlin L. Robb, Nelson L. Michael, Manhattan E. Charurat, Julie Ake, Trevor A. Crowell, Sodsai Tovanabutra. 2019. New Subtype B Containing HIV-1 Circulating Recombinant of sub-Saharan Africa Origin in Nigerian Men Who Have Sex With Men. JAIDS Journal of Acquired Immune Deficiency Syndromes 81:5, 578-584. [Crossref]

4. Erik Billings, Richard A. Heipertz, Tonka Varleva, Eric Sanders-Buell, Anne Marie O'Sullivan, Meera Bose, Shana Howell, Gustavo H. Kijak, Hristo Taskov, Ivailo Elenkov, Marina Nenova, Nedialka Popivanova, Aimee Bolen Valenzuela, Otha Myles, Christian T. Bautista, Merlin L. Robb, Nelson L. Michael, Jerome H. Kim, Paul T. Scott, Sodsai Tovanabutra, Julie A. Ake. 2019. HIV-1 genetic diversity and demographic characteristics in Bulgaria. PLOS ONE 14:5, e0217063. [Crossref]

5. Gustavo H. Kijak, Eric Sanders-Buell, Phuc Pham, Elizabeth A. Harbolick, Celina Oropeza, Anne Marie O’Sullivan, Meera Bose, Charmagne G. Beckett, Mark Milazzo, Merlin L. Robb, Sheila A. Peel, Paul T. Scott, Nelson L. Michael, Adam W. Armstrong, Jerome H. Kim, David M. Brett-Major, Sodsai Tovanabutra. 2019. Next-generation sequencing of HIV-1 single genome amplicons. Biomolecular Detection and Quantification 17, 100080. [Crossref]

6. David Chang, Eric Sanders-Buell, Meera Bose, Anne Marie O'Sullivan, Phuc Pham, Eugene Kroon, Donn J Colby, Rujipas Sirijatuphat, Erik Billings, Suteeraporn Pinyakorn, Nitiya Chomchey, Wiriya Rutvisuttinunt, Gustavo Kijak, Mark Souza, JeanLouis Excler, Praphan Phanuphak, Nittaya Phanuphak, Robert J O'Connell, Jerome H Kim, Merlin L Robb, Nelson L Michael, Jintanat Ananworanich, Sodsai Tovanabutra. 2018. Molecular epidemiology of a primarily MSM acute HIV-1 cohort in Bangkok, Thailand and connections within networks of transmission in Asia. Journal of the International AIDS Society 21:11. . [Crossref]

7. Donn J. Colby, Lydie Trautmann, Suteeraporn Pinyakorn, Louise Leyre, Amélie Pagliuzza, Eugène Kroon, Morgane Rolland, Hiroshi Takata, Supranee Buranapraditkun, Jintana Intasan, Nitiya Chomchey, Roshell Muir, Elias K. Haddad, Sodsai Tovanabutra, Sasiwimol Ubolyam, Diane L. Bolton, Brandie A. Fullmer, Robert J. Gorelick, Lawrence Fox, Trevor A. Crowell, Rapee Trichavaroj, Robert O’Connell, Nicolas Chomont, Jerome H. Kim, Nelson L. Michael, Merlin L. Robb, Nittaya Phanuphak, Jintanat Ananworanich. 2018. Rapid HIV RNA rebound after antiretroviral treatment interruption in persons durably suppressed in Fiebig I acute HIV infection. Nature Medicine 24:7, 923-926. [Crossref]

8. Sung Yong Park, Tanzy M. T. Love, Lucy Reynell, Carl Yu, Tina Manzhu Kang, Kathryn Anastos, Jack DeHovitz, Chenglong Liu, Kord M. Kober, Mardge Cohen, Wendy J. Mack, Ha Youn Lee. 2017. The HIV Genomic Incidence Assay Meets False Recency Rate and Mean Duration of Recency Infection Performance Standards. Scientific Reports 7:1. . [Crossref]

9. Richard A. Heipertz, Ojor Ayemoba, Eric Sanders-Buell, Kultida Poltavee, Phuc Pham, Gustavo H. Kijak, Esther Lei, Meera Bose, Shana Howell, Anne Marie O’Sullivan, Adam Bates, Taylor Cervenka, Janelle Kuroiwa, Akindiran Akintunde, Onyekachukwu Ibezim, Abraham Alabi, Obumneke Okoye, Mark Manak, Jennifer Malia, Sheila Peel, Mohammed Maisaka, Darrell Singer, Robert J. O’Connell, Merlin L. Robb, Jerome H. Kim, Nelson L. Michael, Ogbonnaya Njoku, Sodsai Tovanabutra. 2016. Significant contribution of subtype G to HIV-1 genetic complexity in Nigeria identified by a newly developed subtyping assay specific for subtype G and CRF02_AG. Medicine 95:32, e4346. [Crossref]

10. Laura A. Pacha, Shilpa Hakre, Otha Myles, Eric E. Sanders-Buell, Stephanie L. Scoville, Gustavo H. Kijak, Michael W. Price, Rupal M. Mody, Ying Liu, Shana L. Miller, Phuc T. Pham, Nelson L. Michael, Jerome H. Kim, Sheila A. Peel, Sodsai Tovanabutra, Linda L. Jagodzinski, Steven B. Cersovsky, Paul T. Scott. 2015. Centralized HIV Program Oversight. Medicine 94:46, e2093. [Crossref] 